\title{
Symptoms and Self-Care in Old Age
}

\author{
Joseph M. Holtzman \\ University of Colorado \\ Hiroko Akiyama \\ University of Michigan \\ Alan J. Maxwell \\ Veterans Administration
}

\begin{abstract}
Self-care has recently come to be recognized as the predominant form of response to illness among the general population, but little is known regarding beliefs and practices of the aged. In this study, beliefs regarding the appropriate response to 53 common symptoms were investigated and contrasted to actual responses. Various forms of self-care were seen as appropriate responses to symptoms ranging from minor to potentially serious. Preference for self-care responses appears to be related to perceived severity of symptoms and the perceived effectiveness of available self-care responses.
\end{abstract}

Self-care has recently come to be recognized as the predominant form of response to illness by the population at large. It has been estimated that between $66 \%$ and $85 \%$ of all illnesses are treated through use of self-care measures without recourse to professional assistance (Butler, Gertman, Oberlander, \& Schnidler, 1979-1980; Dean, Holst, \& Wagner, 1983). Studies of self-care practices conducted in Denmark and Great Britain conclude that self-care responses and practices could be considered helpful, effective, or relevant in $62 \%$ to $77 \%$ of instances in which they were used and partially relevant in an additional $12 \%$ of cases (Dean et al., 1983). As Butler et al. (1979-1980) suggest, self-care and self-help hold great promise and potential for the aged.

AUTHORS' NOTE: Address reprint requests to Dr. Holtzman, Department of Applied Dentistry, University of Colorado, School of Dentistry, Box C284, 4200 E. 9th Avenue, Denver, CO 80262.

The Journal of Applied Gerontology, Vol. 5 No. 2, December 1986 183-200

(c) 1987 The Southern Gerontological Society 


\section{Definitions of Self-Care}

Self-care, self-treatment, self-help, self-maintenance, and self-surveillance have frequently been used interchangeably. No broadly accepted definition of what constitutes self-care currently exists. It has been most broadly defined as "an individual's deliberate action on behalf of his own, his family's or his neighbor's well-being"(Butler et al., 1979-1980). Various formulations of self-care have included good nutritional and hygiene practices, common first aid, consumer and patient education, activities of consumer organizations, learned responses to acute and chronic illness, and technical procedures that patients can perform by themselves with proper training (Berg, 1980; Butler et al., 1979-1980). Dean (1981) has identified the self-treatment component of self-care as "decisions by lay persons to diagnose and treat perceived symptoms themselves rather than to seek professional treatment services."

A relatively restrictive definition of self-care was adopted for purposes of this study. We have focused exclusively on behaviors undertaken in response to actual illness or symptoms of illness. Behaviors such as self-maintenance, self-surveillance, and self-help have been excluded. Self-care has been defined as those behaviors, other than consultation with health services providers, that individuals undertake in response to perceived symptoms of physical or mental illness. Selfcare behaviors we considered included self-medication; use of folk or home remedies; changes in activity levels or patterns; and use of other procedures, appliances, and environmental changes undertaken in an attempt to ameliorate symptoms, cure the illness, or cope with associated limitations.

\section{Responses to Symptoms}

Health behaviors of the general population have been extensively studied, particularly in regard to use of professional services. However, relatively little attention has been devoted to systematic studies of self-care responses to specific symptoms. Studies that considered age as an important variable in regard to symptom response have been even rarer.

In an important cross-national study, Andersen, Andersen, and Smedby (1968) found little difference between Swedish and American elders in response to 15 specific symptoms. In both countries, symptom reporting increased with age. Women in both countries reported more 
symptoms than men. Older Americans reported more frequent professional visits than did their Swedish counterparts.

Age was also an important factor in response to illness among federal agency employees studied by Hetherington and Hopkins (1969). Hypersensitivity to symptoms, defined as the propensity to favor physician consultation in response to certain specific symptoms, was noted among widowed, divorced, and married subjects aged 55 and older.

An important series of papers (Brody \& Kleban, 1981, 1983; Brody, Kleban, \& Moles, 1983) focused on symptom experiences and responses of older persons. Working from a list of 20 potentially serious symptoms, Brody and colleagues found that $56 \%$ of experienced symptoms were never reported to health care providers. Older subjects used various forms of self-treatment including use of prescription medications and over-the-counter drugs, and alterations in activity levels and patterns. Approximately $20 \%$ of all symptoms resulted in no specific response.

The meaning individuals attach to symptoms, regardless of age, appears to be an important predictor of the decision to self-treat, ignore, or seek professional help. Banks and Keller (1971) studied responses to 29 specific symptoms. Their findings indicated that persons with previous experience with symptoms expressed less concern and were less likely to seek medical attention. Jones, Wiese, Moore, and Haley (1981) found three underlying factors related to the perceived meaning attached to 45 symptoms: (1) the extent to which the symptoms were perceived as threatening, disruptive, or painful; (2) familiarity of the symptoms and the perceived personal responsibility for their occurrence; and (3) how embarrassing the symptoms were.

Dean and her colleagues (1983) have provided one of the most comprehensive studies of self-care responses to illness. In her study of responses to six common conditions, four major responses were noted, as follows: nonmedicated self-treatment, use of medication, decision to do nothing, and physician consultation that was treated as a form of self-care. Findings included a complex interaction between age, sex, tendency to discuss nonmedical conditions with physicians, and selftreatment. Older persons, particularly women, were found to practice ameliorative responses more frequently than other groups. The reactions of younger people were often directed toward the source of the problem. Neither age nor sex was found to be a significant factor in the decision to ignore a symptom; however there was a direct relationship between age and physician consultation for both men and women. 
Those symptoms normally requiring medication significantly increased with age, although nonmedicated self-treatment was the most commonly practiced response. Such self-treatment included taking vitamins, dressing warmly, staying in bed, using heating pads, avoiding lifting of heavy objects, and, in the case of depression, talking with another person or trying not to think of the underlying problem.

This study builds on the previous work of Brody, Dean, and their colleagues. We are concerned with establishing the course of action that older persons believe to be the most appropriate response to $53 \mathrm{symp}$ toms ranging from minor to potentially serious illnesses. Of special concern is the identification of specific symptoms believed to be most appropriately treated without professional assistance.

\section{Methods}

Based on a comprehensive literature review, consultation with primary care practitioners, and results of a pilot study, we developed a preliminary list consisting of over 200 symptoms commonly experienced by the aged. An effort was made to include symptoms of potentially serious and minor illnesses. The list was then submitted to a panel consisting of an internist, a family practitioner (both directors of their respective departments' geriatrics programs), and a geriatric nurse. The panel was instructed to review the list independently and evaluate the symptoms on the basis of incidence and potential seriousness. Panel members were encouraged to expand the original list to include common symptoms of other illnesses that they felt were absent or to rephrase individual symptoms as necessary. Panel ratings were reviewed and unanimously chosen symptoms were incorporated into a final list. In instances of disagreement, we tried to reach accord through discussions with panel members. Consensus was ultimately achieved on a list of 53 symptoms on which the data were actually collected.

Using the symptom list as a core, we constructed an interview schedule. The schedule incorporated basic demographic information, selfrated health status measures, questions regarding sources of health care, and health problems for which treatment was currently in progress. In response to each symptom, subjects were asked to indicate how serious the symptom should be regarded with responses ranging on a four-point scale from very serious to not serious at all (including a "don't know choice"). Subjects were then asked to select from a list of possible responses the most appropriate response for a person of their own age. 
Choices included the following: see a health professional; take medicine; rest; do nothing (it will go away or is normal); other. When "other" was selected, we asked subjects to specify the type of action. If "take medicine" was selected, we asked subjects to specify prescription, over the counter, folk, or other home remedy. Following a pretest of the interview schedule, trained gerontology center staff conducted interviews in public housing facilities.

\section{Sample}

Subjects, aged 65 and older, were recruited from among residents of 10 publicly owned senior housing facilities in the Oklahoma City area. The sample consisted of 52 Caucasian men, 85 Caucasian women, 41 black women, 6 Native American women, and 2 Hispanic women. Ages ranged from 65 to 91 years with a mean of 72.9. Table 1 summarizes key demographic characteristics.

As might be expected in a group of older persons living independently in community dwellings, relatively few subjects $(15 \%)$ reported their current health status as poor or very poor, with $48 \%$ reporting health as good or very good. In regard to functional health status, $17 \%$ reported physical limitations that prevented them from doing light routine work around the home. While $52.2 \%$ reported inability to perform heavier physical labor (such as scrubbing floors, moving furniture, or lifting heavy objects), $30 \%$ of subjects reported no current limitations in their ability to perform such tasks. $80 \%$ of all subjects reported that their overall health status had either remained the same or actually improved in the previous year.

Although reporting relatively good health status and few serious limitations, the overwhelming majority of subjects $(77.8 \%)$ reported current health problems for which they were either under medical care or taking medication. Of those currently receiving professional care, nearly $95 \%$ reported physicians as the source of that care. Of those taking medication, nearly $92 \%$ reported that these were prescription drugs, another indicator of continuing professional contact.

Thus, the sample on which this study is based can be characterized as noninstitutionalized elderly, predominantly female, and low income. Although the majority were receiving care for one or more health problems at the time of the interview, they reported being relatively well with few serious functional limitations. 
Table 1. Study Participants: Selected Characteristics

\begin{tabular}{|c|c|c|}
\hline & Range & Mean \\
\hline \multicolumn{3}{|l|}{ Age } \\
\hline Male & $65-88$ & 72.9 \\
\hline \multirow[t]{2}{*}{ Female } & $65-96$ & 72.8 \\
\hline & $N$ & $\%$ \\
\hline \multicolumn{3}{|l|}{ Sex } \\
\hline Male & 52 & 28.0 \\
\hline Female & 134 & 72.0 \\
\hline \multicolumn{3}{|l|}{ Race } \\
\hline Caucasian & 137 & 73.7 \\
\hline Black & 41 & 22.0 \\
\hline Other & 8 & 4.3 \\
\hline \multicolumn{3}{|l|}{ Marital status } \\
\hline Married & 47 & 25.1 \\
\hline Widowed & 102 & 54.6 \\
\hline Separated/divorced & 33 & 17.7 \\
\hline Never married & 5 & 2.7 \\
\hline \multicolumn{3}{|l|}{ Annual income } \\
\hline $0-4999$ & 103 & 57.9 \\
\hline $5000-9999$ & 40 & 22.5 \\
\hline Over 9999 & 35 & 19.7 \\
\hline \multicolumn{3}{|c|}{ Formal education in years } \\
\hline $0-6$ & 29 & 15.8 \\
\hline $7-12$ & 124 & 67.8 \\
\hline Over 12 & 30 & 16.4 \\
\hline \multicolumn{3}{|l|}{ Religious affiliation } \\
\hline Protestant & 151 & 81.2 \\
\hline Catholic & 24 & 12.9 \\
\hline Other/none & 10 & 5.4 \\
\hline
\end{tabular}

NOTE: Percentages may not equal 100 due to rounding.

\section{Beliefs Regarding Symptom Seriousness}

Virtually all of the symptoms studied could be related to potentially serious physical or mental illnesses in this age group, although this is clearly more likely for some symptoms (unexplained bleeding) than for others (dry skin). Participants in this study believed in the potential seriousness of symptoms: Of the 53 symptoms studied, 43 were classified as "serious" or "very serious" by $50 \%$ or more (see Table 2 ).

Although clearly skewed toward the belief that most symptoms should be regarded as serious, older persons were not indiscriminate in the relative importance they attached to various symptoms. Symptoms 


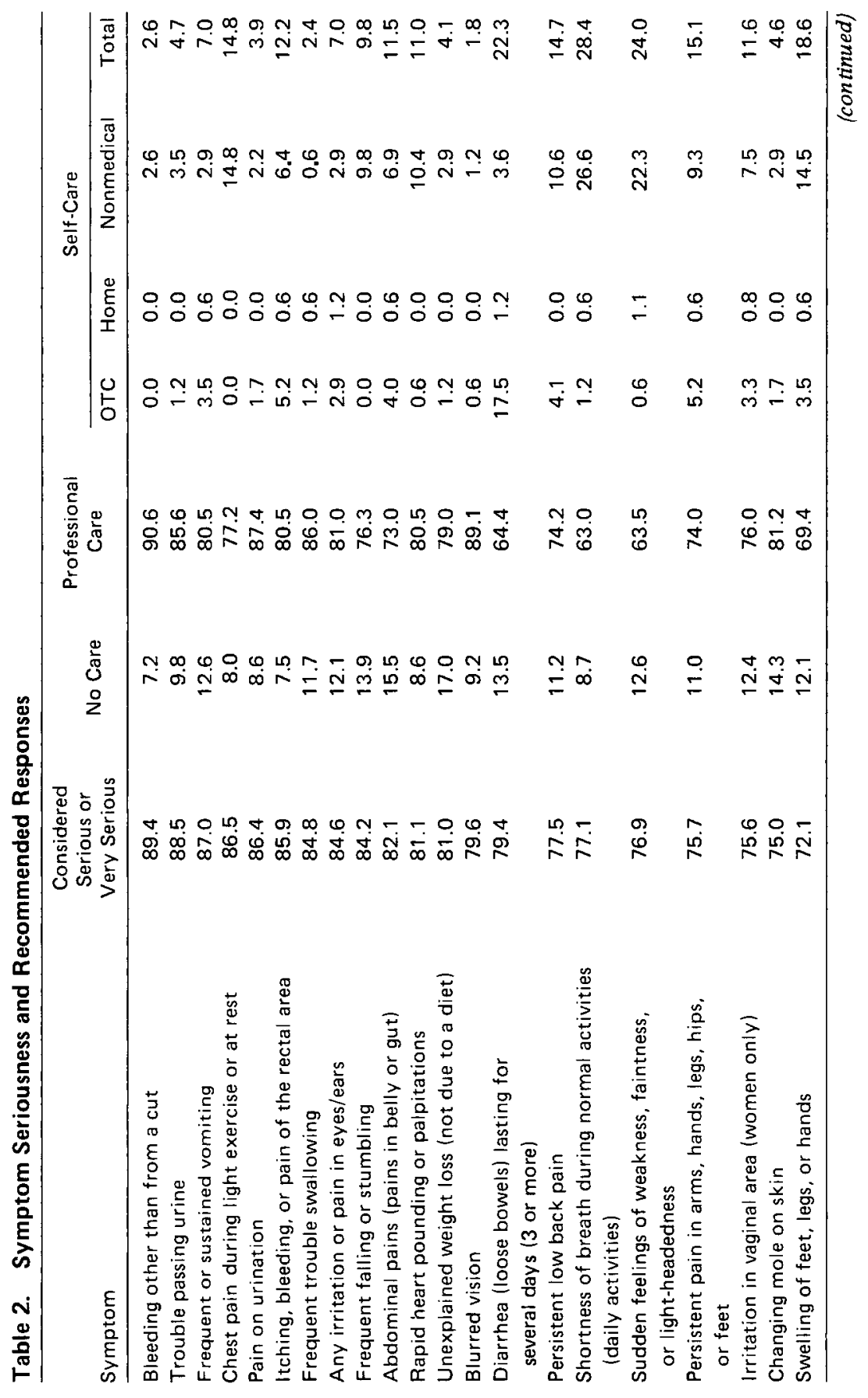




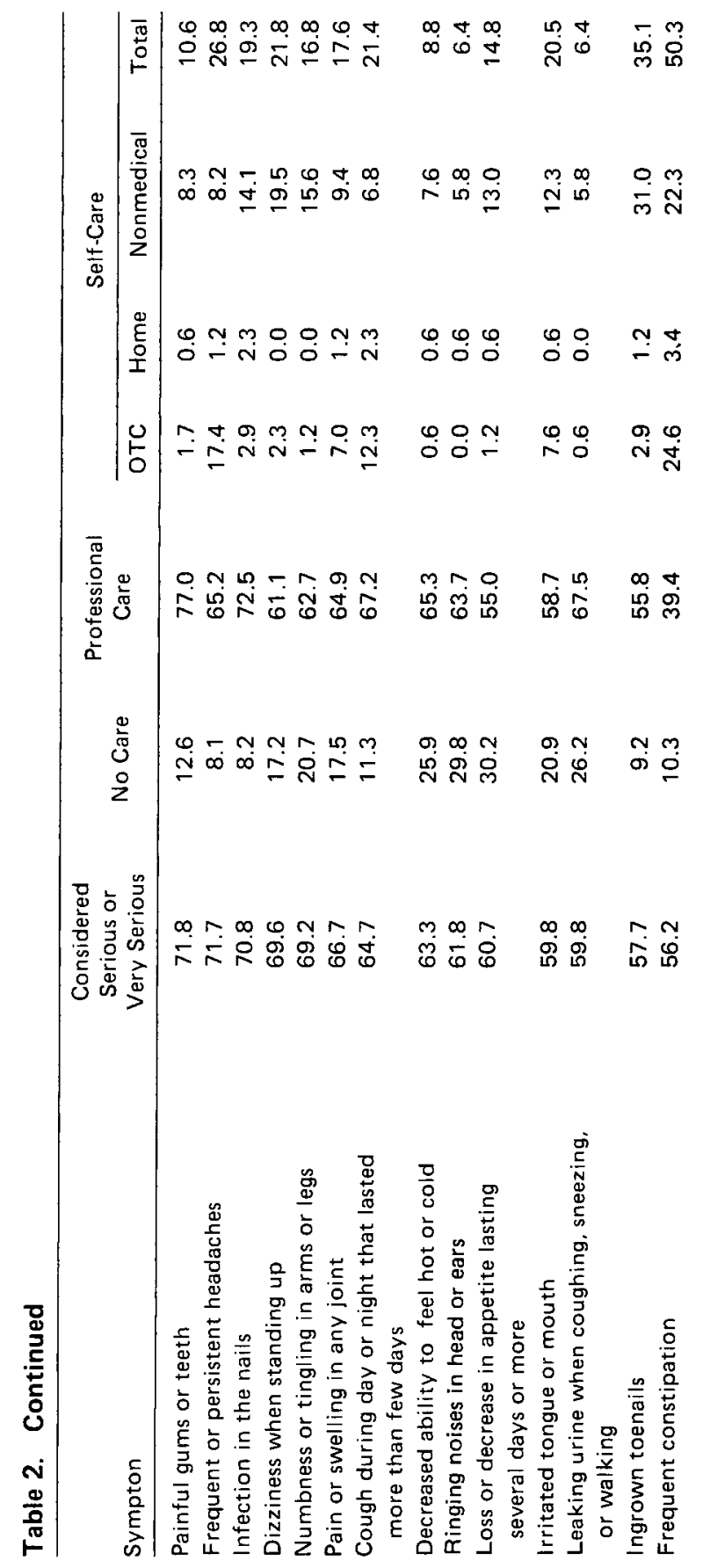




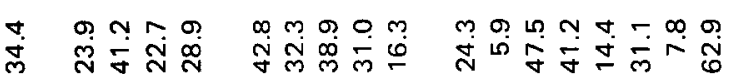

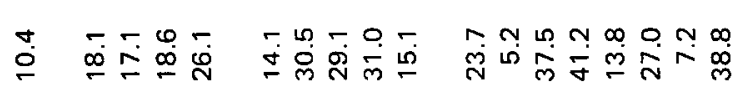

$\begin{array}{llllllllllllll}0 & 0 & 0 & 0 & m & 0 & 0 & 0 & 0 & 0 & 0 & 0 & 0 & 0\end{array}$

๙

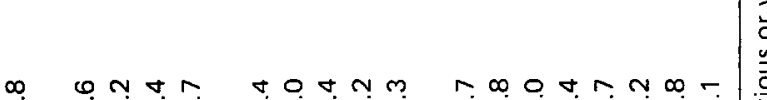

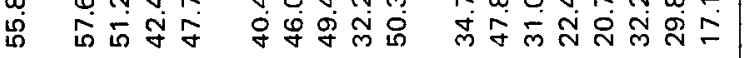

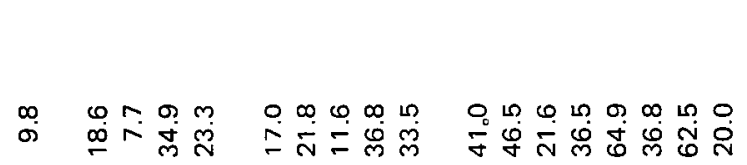

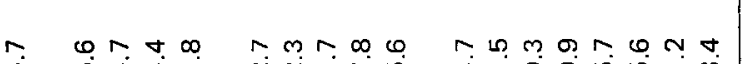

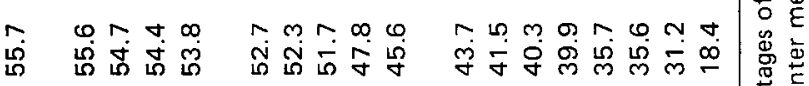

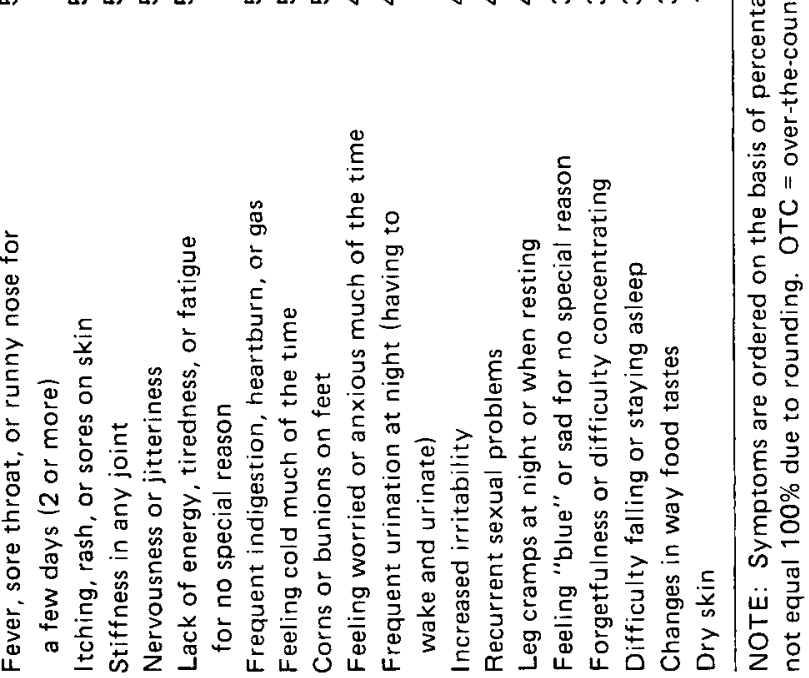


that were painful, unusually persistent, inexplicable, or potentially disruptive of daily routines tended to be ranked more seriously than symptoms that might be regarded as commonplace (dry skin, leg cramps, corns, stiffness). Table 2 provides rankings of symptoms on the basis of the relative seriousness attributed to them by study participants.

Particularly striking was the lack of importance attached to symptoms of possible mental illness as compared to symptoms of physical illness. Of the 10 symptoms regarded least serious by the subjects, four are clearly more consistent with mental than physical illness. These include feeling worried or anxious much of the time, increased irritability, forgetfulness or difficulty concentrating, and feeling "blue" or sad for no specific reason. When "recurrent sexual problems" and "difficulty falling or staying asleep" (both frequently attributed to psychogenic causes) are included, 6 of the least seriously regarded 10 symptoms fall into this category. These older respondents did not regard psychological problems with the same degree of concern attributed to symptoms of physical illness. They did not recognize these symptoms as potentially indicative of serious illness.

Race and sex differences in significance attached to symptoms were evaluated and have been previously reported (Holtzman \& Akiyama, 1985). Although some significant differences were observed between whites and blacks and between men and women, overall levels of agreement were moderate to high ranging from .56 to .74 (Kendall's tau b, $\mathrm{p}=.0001)$.

\section{Seriousness and Appropriate Responses}

It has frequently been suggested that perceived symptom seriousness is an important determinant of the decision to seek professional care. When subjects were asked to select the most appropriate response to each symptom for a person their own age, we observed a moderate to strong relationship between perceived seriousness and the recommendation to seek professional care (Kendall's tau $b=.71, p=.0001$ ). Although some significant differences were observed by sex and race, the overall picture was one of a consistently positive relationship between perceived seriousness and perceived need to seek professional care. The more seriously a symptom was perceived, the more likely was the recommendation for professional care as the appropriate response (Holtzman \& Akiyama, 1985). 
Respondents in this study saw self-care as an appropriate alternative to use of professional services in response to a diverse group of symptoms. The percentage of respondents recommending self-care ranged from $62.9 \%$ in response to dry skin to $1.7 \%$ in response to blurred vision (Table 2). Symptoms such as constipation, leg cramps, indigestion, feeling "blue" or sad, and corns or bunions on the feet were seen as particularly amenable to one or another form of self-care.

Generally speaking, an inverse relationship was found to exist between perceived symptom seriousness and the recommendation of self-care as the most appropriate response. Thus, for example, symptoms such as bleeding other than from a cut, blurred vision, and pain on urination, all regarded as very serious, were rarely seen as appropriate for self-care. However, symptoms ranked seriously or very seriously by high percentages of elders were not always seen as inappropriate for self-care. Symptoms such as constipaton, fever, sore throat, or runny nose for a few days, and frequent or persistent headaches were viewed as potentially serious, although appropriately treated through self-care by substantial numbers of respondents. The ready availability of over-thecounter drugs believed to be effective in treatment of these symptoms probably accounts for the relative willingness to recommend self-care in the face of symptoms believed to be serious by substantial numbers of the elderly.

It should be noted that symptoms ranked as relatively nonserious were not always seen as amenable to self-care. For example, although changes in the way food tastes, recurrent sexual problems, and nervousness or jitteriness were regarded as relatively not serious, they were rarely seen as treatable without recourse to professional care. The perception of availability, or perhaps the lack of availability of effective means of self-treatment, or the attribution of these symptoms to normal changes associated with aging, may account for recommendations of professional care or no active response.

\section{Actual Responses to Symptoms}

Subjects were asked to report which, if any, of the symptoms they had experienced in the previous 12 months. Those reporting a symptom experience were asked to indicate what course of action they had taken in response, selecting from the same group of responses previously used. A total of 1,392 symptom experiences were thus elicited. All 53 symp- 
toms were reported to have been experienced by one or more subjects; frequency ranged from 60 for leg cramps to 3 for unexplained weight loss.

Findings discussed above indicated that the overwhelming majority of symptoms were regarded as serious or very serious and that seeking professional services was seen as the most appropriate response to most symptoms. However, an analysis of reported responses to actual symptoms revealed that these responses rarely mirrored precisely the recommended responses of the elders themselves. Actual use of professional services never approached levels of use which might be predicted on the basis of subjects' perceptions of seriousness or beliefs regarding appropriate responses (Table 3).

As might be expected, older persons simply ignored substantial numbers of symptoms (no active response taken). For example, while $69.6 \%$ of subjects recognized dizziness as serious or very serious, fewer than $17 \%$ of the 42 who experienced it sought professional help. The majority (64.3\%) chose simply to ignore it. Similarly, of the 50 subjects reporting shortness of breath, $46 \%$ ignored the symptom. Psychobehavioral disturbances such as "feeling blue"; feeling worried, nervous, or jittery; and forgetfulness were similarly most frequently ignored.

Close observation of reported responses reveals that instances of use of various forms of self-care in response to symptoms regarded as serious were considerably more frequent than might have been expected on the basis of beliefs regarding appropriate responses. For example, substantial percentages of subjects reported self-care responses to symptoms such as abdominal pain, shortness of breath, persistent headaches, and ingrown toenails although use of professional services was seen as the most appropriate response. Although actual numbers of subjects reporting symptoms experiences were small, symptoms of potentially very serious illness such as chest pain, sudden feelings of weakness, and frequent falling or stumbling were reportedly self-treated by substantial percentages of subjects experiencing them.

Self-care responses to symptoms varied widely. Use of over-thecounter crugs, vitamins, minerals, and other substances (e.g., Vaseline and moisturizing creams) were quite common in response to symptoms and conditions for which they are frequently advertised and sold. For example, many elderly people used aspirin, Tylenol, and related drugs for treatment of fever, persistent headaches, and joint stiffness. Drugs such as Rolaids, Tums, Maalox, and other antacids were used for 


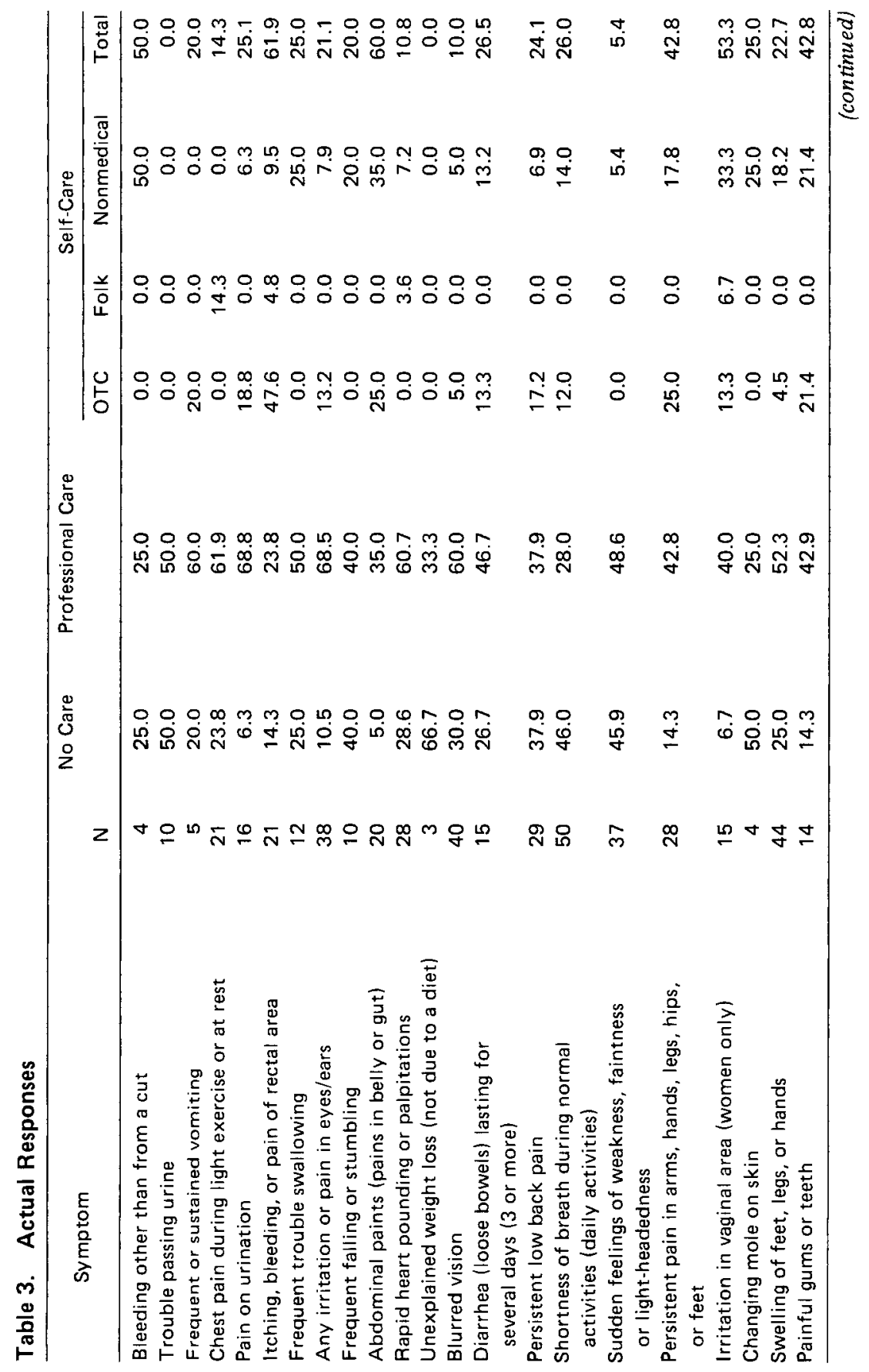




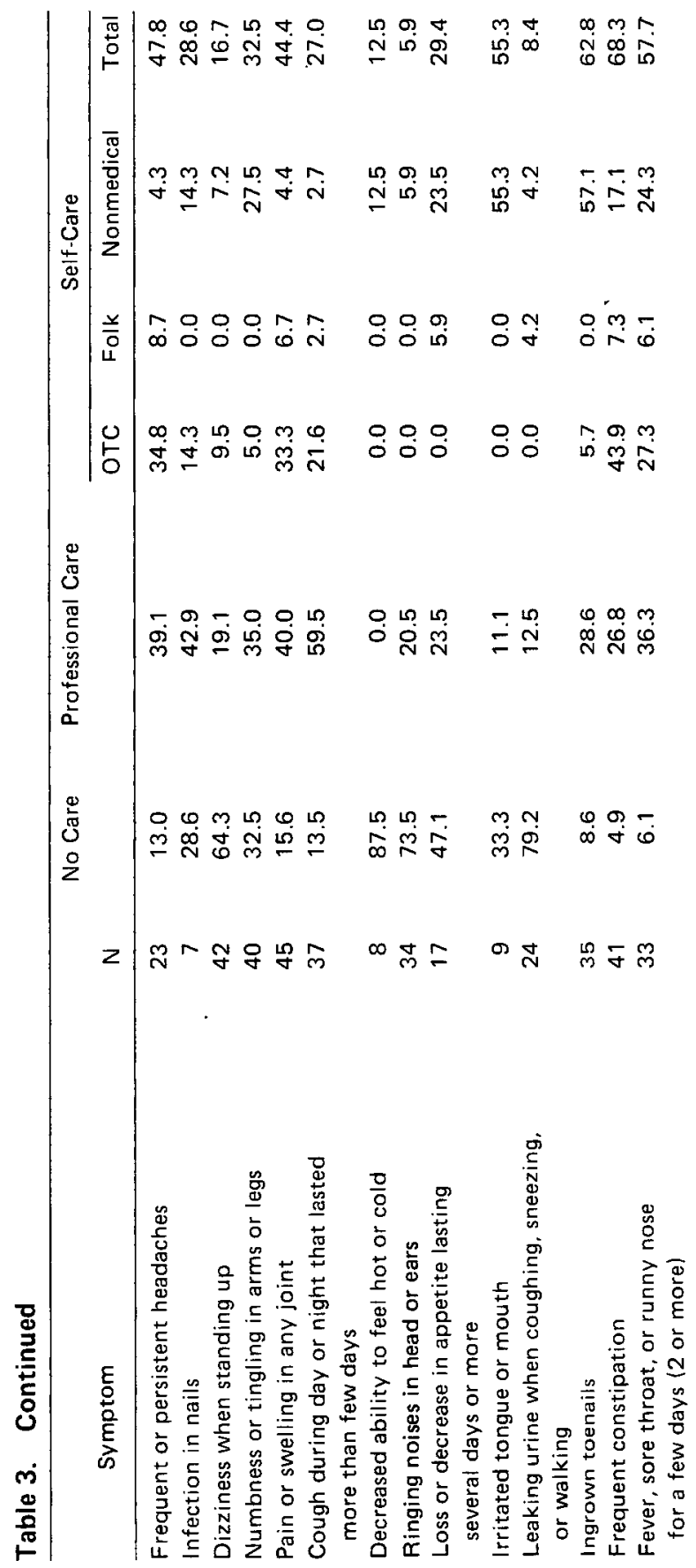




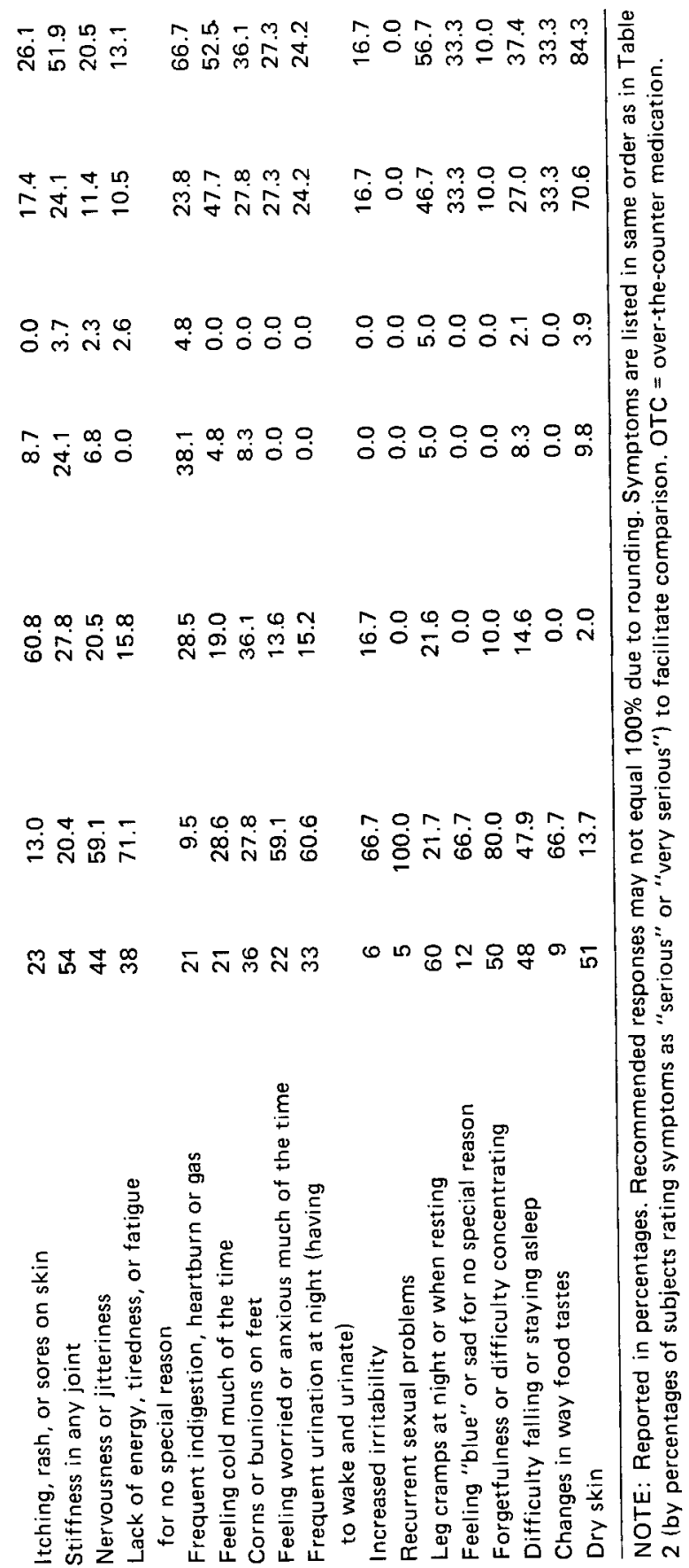


indigestion and various laxatives for constipation. Skin creams and lotions were applied for treatment of dry skin.

Occasionally, drugs and other over-the-counter substances were used in nonstandard ways. For example, vitamins were used for numbness or tingling in arms and legs, chest pain, and leg cramps. However, the frequency of inappropriate or potentially dangerous use of over-thecounter drugs was low in response to individual symptoms. Generally speaking, elders who used over-the-counter drugs and related substances did so for minor or commonplace symptoms. They used these drugs and other substances for the purposes for which they had been advertised and commonly believed to be effective.

Although rare in this population, use of home or folk remedies for treatment of various symptoms was reported. Substances such as camphor, vinegar and water, and lemon juice and honey were used for a variety of ailments. But few subjects used such home remedies or folk medicine, even in response to relatively minor symptoms (Table 3 ). This relative rarity of use of such remedies may be a function of the ready availability of over-the-counter medications and the urban nature of the population studied.

The category "nonmedicated self-care" includes the broadest range of behaviors reported. Responses ranged from alterations in life-style such as exercise and dietary patterns, and the development of psychological coping mechanisms, to application of simple mechanical techniques. Among the simplest response behaviors were toward feeling cold much of the time. Typical responses included wearing warmer clothing, turning up the heat, and making other alterations in the living environment. Reducing consumption of liquids was a common response to frequent urination and leaking urine. Some symptoms drew conflicting responses. For example, the treatment of dry skin and itching and rash or sores on the skin included both increased and decreased bathing.

Reduction in level of physical activity was another frequent "nonmedicated" response to actual symptoms. Symptoms such as lack of energy, shortness of breath, chest pain, and sudden feelings of weakness were often treated with rest. Behaviors included sitting down for rest periods, trying to get more sleep, and less frequently engaging in strenuous activities.

\section{Conclusion}

The recent intensification of efforts to control costs of medical care coupled with continuing consumer dissatisfaction with some aspects of 
current professional practice have set the stage for a rediscovery of self-care. Health care professionals have themselves begun to recognize that some forms of self-care are appropriate as useful adjuncts to professional services. However, before self-care can fulfill its promise and potential, more must be known about current self-care practices of the aged, the symptoms they believe can be appropriately treated, and their willingness to follow self-care regimens.

This study, although limited by a relatively small and homogenous sample, does provide evidence that self-care responses are common at least as a preliminary mode of response to a broad range of symptoms among the aged. This finding is consistent with earlier work conducted in Denmark, England, and the United States that focused primarily on members of younger age groups using more limited symptom lists.

Our findings do not suggest that self-care responses are always appropriate, effective, or even safe. Indeed, we observed potentially dangerous self-care responses by a small number of the aged in regard to symptoms of potentially life-threatening illnesses. However, as previous researchers have suggested, self-care responses by this group of elderly participants tended to be appropriate, potentially effective, or at least benign in the overwhelming majority of instances.

Further, although not specifically investigated, the findings suggest that the selection of self-care responses actually reported appears to be related to the availability of drugs, appliances, and procedures believed to be effective. Note that where over-the-counter drugs useful in symptomatic relief were available, they were frequently the response of choice. Similarly, when simple environmental or behavioral changes were possible, there was a willingness to use them prior to seeking professional care. Examination of second responses and associated comments indicates that use of professional services became the response of choice when self-care procedures failed to produce the desired result, or in instances in which symptoms persisted or became more severe.

Our findings indicate the widespread practice of self-care among the aged. With the wider availability of data on current beliefs and practices in regard to specific symptoms, it should be possible to design training materials and programs to ensure that responses will be appropriate and effective. Similarly, health care professionals need to become aware of specific symptoms that the aged may not report, dismiss as unimportant, or treat inappropriately. Questions concerning these symptoms should become a routine part of history taking, and should be raised during the investigation of presenting complaints. 


\section{References}

Andersen, M., Anderson, O., \& Smedby, B. (1968). Perception of a response to symptoms of illness in Sweden and the United States. Medical Care, 6, 18-30.

Banks, F., \& Keller, M. (1971). Symptom experience and health action. Medical Care, 9, 498-502.

Berg, A. (1980). Targeting symptoms for self-care health education: A multi-variate analysis of physician contacts. Medical Care, 18, 551-555.

Brody, E., \& Kleban, M. (1981). Physical and mental health symptoms of older people: Who do they tell? Journal of the American Geriatrics Society, 29, 442-449.

Brody, E., \& Kleban, M. (1983). Day-to-day mental and physical health symptoms of older people: A report on health logs. Gerontologist, 23, 75-85.

Brody, E., Kleban, M., \& Moles, E. (1983). What older people do about their day-to-day mental and physical health problems. Journal of the American Geriatrics Society, 31, 489-498.

Butler, R., Gertman, J., Oberlander, D., \& Schnidler, L. (1979-1980). Self-care, self-help, and the elderly. International Journal of Aging and Human Development, 10, 95-119.

Dean, K. (1981). Self-care responses to illness: A selected review. Social Sciences and Medicine, 21, 1012-1032.

Dean, K., Holst, E., \& Wagner, M. (1983). Self-care of common illnesses in Denmark. Medical Care, 21, 1012-1032.

Hetherington, R., \& Hopkins, C. (1969). Symptom sensitivity: Its social and cultural correlates. Health Services Research, 4, 63-75.

Holtzman, J., \& Akiyama, A. (1985). Symptoms and the decision to seek professional care. Gerodontics, I, 44-49.

Jones, R., Wiese, H., Moore, R., \& Haley, J. (1981). On the perceived meaning of symptoms. Medical Care, 19, 710-717.

Joseph M. Holtzman, Ph.D., is Associate Professor of Applied Dentistry and Research Professor of Gerodontics at the University of Colorado School of Dentistry. His current research focuses on cross-national comparisons of perceptions and response to oral health symptoms in the aged.

Hiroko Akiyama, Ph.D., is a Research Fellow in the School of Social Work at the University of Michigan, Ann Arbor.

Alan J. Maxwell, M.D., is Chief of Extended Care at the Veterans Administration Medical Center in Tuscaloosa, Alabama. 